\title{
[11C]-Methionine PET for Identification of Pediatric High-Grade Glioma
}

\section{Recurrence}

${ }^{1}$ Asim K. Bag, MD; ${ }^{1}$ Melissa N. Wing, ${ }^{1}$ Noah D. Sabin, MD; ${ }^{1}$ Scott N. Hwang, MD; ${ }^{2}$ Gregory T. Armstrong, MD, MSCE; ${ }^{3}$ Yuanyuan Han, PhD; ${ }^{3}$ Yimei Li, PhD, ${ }^{1}$ Scott E. Snyder, PhD; ${ }^{4}$ Giles W.

Robinson, MD; ${ }^{4,5}$ Ibrahim Qaddoumi, MD, MS; ${ }^{4}$ Alberto Broniscer, MD; ${ }^{6}$ John T. Lucas and ${ }^{1}$ Barry L. Shulkin.

${ }^{1}$ Department of Diagnostic Imaging, St. Jude Children's Research Hospital, Memphis, Tennessee, USA

${ }^{2}$ Department of Epidemiology and Cancer Control, St. Jude Children's Research Hospital, Memphis, Tennessee, USA

${ }^{3}$ Department of Biostatistics, St. Jude Children’s Research Hospital, Memphis, Tennessee, USA

${ }^{4}$ Department of Oncology, St. Jude Children's Research Hospital, Memphis, Tennessee, USA

${ }^{5}$ Department of Global Pediatric Medicine, St. Jude Children's Research Hospital, Memphis, Tennessee, USA

${ }^{6}$ Department of Radiation Oncology, St. Jude Children's Research Hospital, Memphis, Tennessee, USA

\section{Corresponding Author:}

Asim K. Bag

Associate Member, Department of Diagnostic Imaging

262 Danny Thomas Place, Mail Stop 220, Memphis, TN 38105

Tel: 901.595.3347; Fax: 901-595-3981

E-mail: asim.bag@stjude.org

Running title: [11C]MET-PET for pediatric high-grade glioma

Disclaimer: None

Abstract Word Count: 296

Main Text Word Count: 3600 


\begin{abstract}
Rationale: Differentiating tumor recurrence or progression from pseudoprogression during surveillance of pediatric high-grade gliomas (PHGGs) using magnetic resonance imaging (MRI), the primary imaging modality for evaluation of brain tumors, can be challenging. The aim of this study was to evaluate whether [11]Cmethionine positron emission tomography ([11C]MET -PET), a molecular imaging technique that detects functionally active tumors, is useful for further evaluating MRI changes concerning for tumor recurrence during routine surveillance.
\end{abstract}

Methods: We evaluated 27 lesions in 26 patients with new or worsening MRI abnormalities, where PHGG tumor recurrence was of concern during follow-up visits with [11C]MET -PET. We performed quantitative and qualitative assessments of both [11C]MET-PET and MRI data to predict the presence of tumor recurrence. Further, to assess for an association with overall survival we plotted the time from development of the imaging changes against survival.

Results: Qualitative evaluation of [11C]MET-PET achieved 100\% sensitivity, 60\% specificity, and 93\% accuracy to correctly predict the presence of tumors in 27 new or worsening MRI abnormalities. Qualitative MRI evaluation achieved sensitivity ranging from $86 \%$ to $95 \%$, specificity ranging from $40 \%$ to $60 \%$, and accuracy ranging from $85 \%$ to $89 \%$. The interobserver agreement for [11C]MET-PET assessment was $100 \%$, whereas the interobserver agreement was only 50\% for MRI $(P=<0.01)$. Quantitative MRI and [11C]MET-PET evaluation using receiver operating characteristics demonstrated higher specificity $(80 \%)$ than qualitative evaluations (4060\%). Postcontrast enhancement volume, metabolic tumor volume, tumor-to-brain ratio and presence of tumor as determined by consensus MRI assessment were inversely associated with overall survival.

Conclusion: [11C]MET-PET has slightly higher sensitivity, and accuracy for correctly predicting presence of tumor recurrence, with excellent interobserver agreement, than does MRI. Quantitative [11C]MET-PET can also predict overall survival. These findings suggest [11C]MET-PET can be useful for further evaluation of MRI changes during surveillance of previously treated PHGG.

Key Words: MRI; [11C]MET-PET; pediatric high-grade glioma; pseudoprogression; recurrence. 


\section{INTRODUCTION}

It has only recently been discovered that pedatric high-grade gliomas (PHGGs) are biologically distinct from adult high-grade gliomas (1). However, this new knowledge has not yet changed diagnoses, classifications, World Health Organization (WHO) grading, or treatment of PHGGs (2). PHGGs in children older than 3 years are treated with a combination of maximal safe surgical resection, radiation therapy with or without adjuvant chemotherapy, and subsequent continued chemotherapy, similar to the treatment regimen for adult high-grade gliomas (3-5). Despite this aggressive therapy, outcomes in young children are dismal, with a local 1-year failure-free survival rate of 60\% (6), suggesting that recurrence is common. Accurate diagnosis of tumor recurrence is important because a) the median overall survival (OS) of recurrent PHGGs is 4 to 7 months (7) and b) treatment of pseudoprogression is different from that of tumor recurrence. However, the diagnosis of recurrence is not always straightforward with magnetic resonance imaging (MRI), which is the clinical standard of care test for assessing responses to treatments. Indeed, treatment-related effects including pseudoprogression frequently mimics tumor recurrence, thereby leading to misdiagnosis and incorrect management $(8,9)$.

Pseudoprogression is characterized by temporary enlargement and increased enhancement of clinical target volumes with MRI (10) and occurs in up to $20 \%$ of patients treated with radiation therapy and adjuvant chemotherapy (11). The incidence of pseudoprogression following initial therapy of PHGGs is smiliar to the incidence in adults following treatment of high grade gliomas (12). Tumor recurrence is also characterized by enlargement of tumor volume with increased enhancement making the distinction challenging (13-15). Many advanced MRI techniques have been extensively studied to differentiate treatment-related effects from true tumor progression with variable benefits (16-19). Positron emission tomography with various radiotracers has been studied to distinguish true tumor progression from 
pseudoprogression $(17,20-24)$. Of the many PET radiotracers used to evaluate tumor recurrence, in adults, study results using amino acid PET treacers (i.e., [11C]-methionine ([11C]MET), [18F] Fluoroethyl tyrosine (FET) and [18F] - Dihydroxyphenylalanine (F-DOPA)) suggests that that a reduction of amino acid uptake and/or a decrease of the metabolically active tumor volume is a sign of treatment response associated with long-term outcome ${ }^{25}$. Response assessment in Neuro-Oncology working group and European Association for Neuro-Oncology now suggest that [18F]-FET may facilitate the diagnosis of pseudoprogression in glioblastoma patients within the first 12 weeks following completion of chemoradiotherapy (25). [11C]MET, a true amino acid PET tracer with properties very similar to 18 FET PET, has recently been shown to differentiate true tumor progression from treatmentrelated effects than other PET tracers in adults, with a sensitivity and specificity of $91.2 \%$ and $87.5 \%$, respectively (26). Although the utility of [11C]MET-PET for evaluating nonenhancing PHGGs has been investigated (27), it has not been systematically investigated to evaluate tumor recurrence in PHGGs.

Here, we evaluated whether [11C]MET-PET can be useful for the identification of tumor recurrence in previously treated PHGGs. Specifically, we compared the accuracy of [11C]MET-PET for predicting the presence of tumors when recurrence is suspected with that of MRI. We also compared the interobserver agreement of [11C]MET-PET and MRI to determine whether [11C]MET-PET imaging adds value to conventional MRI and whether [11C]MET-PET or MRI can predict overall survival (OS).

\section{MATERIALS AND METHODS}

\section{Study subjects}

We retrospectively included all subjects with PHGGs who were enrolled in the ongoing "Methionine PET/CT studies in patients with cancer" clinical trial (NCT00840047) at St. Jude Children's Research Hospital (St. Jude) since 2009. This study was approved by the St. Jude Institutional Review Board and each subject, or parent or legal gurdian signed informed consent to participate. The inclusion criteria for this study were as follows: (1) 
previously treated WHO grade III or IV PHGGs that demonstrated worsening or new imaging abnormalities on fluid-attenuated inversion recovery (FLAIR) sequences, or on postcontrast T1-weighted sequences, or on both the sequences during routine surveillance MRIs, in comparison with the MRI findings from the baseline or from the best response; (2) [11C]MET-PET scans obtained within 3 weeks of the surveillance MRI scans; and (3) definitive diagnosis of tumor recurrence was established within 8 weeks of either the MRI surveillance scan or [11C]MET-PET scan.

\section{Imaging acquisition}

\section{[11C]MET-PET}

[11C]MET was prepared as previously described (28). [11C]MET-PET imaging occured after at least 4 hours of fasting. Each subject received intravenous injections of $740 \mathrm{MBq}(20 \mathrm{mCi})$ of [11C]MET per $1.7 \mathrm{~m}^{2}$ of body surface area (maximum prescribed dose, $740 \mathrm{MBq}$ ). Transmission computed tomography (CT) images for attenuation correction and lesion localization and PET images were acquired approximately 5-15 (8.7 \pm 3.3$)$ minutes after [11C]MET injection with a GE Healthcare Discovery 690 PET/CT scanner or GE Discovery LS PET/CT scanner (GE Healthcare) using these parameters: field-of-view $=30 \mathrm{~cm}$; matrix $=192 \times 192$; recon method $=$ VUE point HD; quantification method = SharpIR; Filter cutoff $=5.0 \mathrm{~mm}$; subsets $=34$; iterations $=4$; z-axis filter $=$ standrd. Q.Clear350-SharpIR quantification method was used only in one subject. The CT acquisition parameters are as

follows: $0.5 \mathrm{~cm}$ slice thickness, $0.8 \mathrm{~s}$ tube rotation, $1.5 \mathrm{~cm} /$ rotation table speed, $1.5: 1$ pitch, $120 \mathrm{kV}$, and $90 \mathrm{~mA}$ with dose modulation. PET images were acquired in 3D mode for $15 \mathrm{~min}$. Data were reconstructed into multiplanar cross-sectional images with standard vendor-supplied software and displayed on a nuclear medicine workstation (Hermes Medical Systems, Inc.) for analysis. 
The following sequences were acquired with a 1.5 Avanto magnet or 3T TrioTim, Skyra, or Prisma magnet (Siemens Medical Solutions) with $0.1 \mathrm{mmol} / \mathrm{Kg}$ intravenous gadobutrol (Gadavist, Bayer Health Care): 3D magnetization-prepared rapid gradient echo $\left(1 \mathrm{~mm}^{3}\right.$ isotropic acqusition, $1590 \mathrm{~ms}$ repetition time [TR], $2.7 \mathrm{~ms}$ echo time [TE], $900 \mathrm{~ms}$ inversion time [TI], and $15^{\circ}$ flip angle [FA]); 2D transverse T1 fast low angle shot (4-mm slice thickness [ST], no gap, $259 \mathrm{~ms}$ TR, $2.46 \mathrm{~ms} \mathrm{TE}$, and 70 $\mathrm{FA}$ ); 2D transverse diffusion-weighted sequence and postcontrast 2D transverse $\mathrm{T} 1$ flash low angle shot (parameters identical with those of precontrast axial 2D T1); 2D transverse T2 TSE (4mm ST, no gap, $4810 \mathrm{~ms}$ TR, $87 \mathrm{~ms} \mathrm{TE}$, and 180 ${ }^{\circ} \mathrm{FA}$ ); 2D transverse T2 FLAIR (4-mm ST, no gap, 10000 ms TR, 106 ms TE, $2600 \mathrm{~ms} \mathrm{TI}$, and 130 FA); and 3D sagittal T1 (parameters identical with those of precontrast sagittal 3D T1). Apparent diffusion coefficient maps were calculated from the diffusion images with the vendor provided software (Syngo, Siemens Healthcare).

\section{Qualitative image analysis}

\section{MRI}

Each surveillance MRI was evaluted four times. The first evaluation was performed during generation of the clinical report by one of the neuroradiologists assigned to the clinical service. The second evaluation was performed by a single neuroradiologist (NDS, observer 1) with twelve years experience evaluating response assessments of pediatric brain tumors. The third evaluation was performed by a single neuroradiologist (SNH, observer 2) with eight years experience evaluating response assessments of pediatric brain tumors. Both observers were blinded to the [11C]MET-PET findings and did not have access to any clinical information or any imaging studies obtained after the index surveillance MRI. The fourth evaluation consisted of a consensus evalauation by observers 1 and 2. New or worsening MRI abnormalities were subjectively categorized as definitely tumor (score $=1$ ), definitely not tumor ( score $=2$ ), or indeterminate (score $=3$ ). The consensus readings were also scored with the same 1-3 scale. If a discrepancy in opinion occurred between two observers, the reading was scored as 3 . The first rating from neuroradiologists on clinical duties was scored with the same scale on the basis of the clinical reports. Diffusion and apparent diffusion coefficient maps were used together for subjective evaluation only. 


\section{[11C]MET-PET}

[11C]MET-PET images were independently reviewed by 2 observers, 1 with 15 years experience (BLS) and the other with 2 years' experience (AKB) in molecular imaging for assessments of treatment response in pediatric brain tumors. The observers were provided the location of the MRI abnormality with access to the MRI images. The [11C]MET-PET images were rated qualitatively on a 4-point scale relative to frontal white matter (in all of the included subjects at least some component of the frontal lobe white matter was free of tumor) : 0: no detectable uptake, or 1: mild uptake but less than contalateral frontal lobe white matter, or 2: mild uptake similar to the contralateral frontal lobe white matter, or 3: uptake greater than frontal lobe white matter. Finally, the results of visual assessment were consolidated into just two groups. The first group was "no or same or lower uptake than the reference region (grades 0,1 , and 2) and the second group was "higher uptake than the reference region (grade 3)".

\section{Quantitative imaging analysis}

Worsening or new imaging abnormalities on postcontrast T2 FLAIR and T1-weighted sequences were manually segmented by using Vitrea Advanced Visualization (Vital Images) software. Three patients had subtle enhancement on T1-weighted sequences, and their T1-weighted regions of interest were drawn on the delta T1 images (precontrat T1-weighted images were subtracted on a voxel-by-voxel basis from the postcontrast T1weighted images).

Standardized uptake values (SUV) for the [11C]MET-PET images were calculated using Hermes software. After co-registration of the PET data set with FLAIR and/or postcontrast T1 weighted MRI sequences, regions of interest were manually drawn either around the areas of abnormal [11C]MET uptake or around the MRI abnormality. In addition, quantitative tumor metrices (metabolic tumor volume, MTV; tumor to brain ratio, TBR) were calculated as suggested by Laws et al (29). However, instead of using a cresecentic ROI,we used a $1.0 \mathrm{~cm}^{3}$ sphere to calculate SUVmean of the contralateral normal prefrontal lobe cortex and juxtacortical white matteras as suggested by Hotta et al (22) for consistency. Briefly, was calculated using. The three-dimensional metabolic 
tumor volume with an SUV >1.3-times greater than normal brain cortex (obtained on the prior step) was automatically contoured using Hermes software, which automatically calculated the SUVmax, and SUVmean of the tumor. Tumor-to-brain ratios (TBR: TBRmax) were then manually calculated by dividing the tumor SUVMax with the SUVmean of the contralateral normal frontal lobe cortex. TBRmean was manually calculated by dividing the tumor SUVmean with the SUVmean of the contralateral normal frontal lobe cortex. In lesions with SUV of $<1.3$ times the contralateral frontal lobe, a volume of interest (VOI) was manually drawn on the FLAIR abnormal areas and agreed upon by both nuclear medicine physicians and then the VOIs were cpied to the PET images. The SUVmax of the VOIs were autimatically calculated by the software. The TBR was then calculated as described above.

\section{Final outcomes}

The final outcomes of the lesions evaluated with MRI and [11C]MET-PET were determined with the following methods: (1) response assessment in neuro-oncology criteria applied to imaging and clinical findings; (30) (2) biopsies; or (3) follow-up imaging and clinical course. Tumor was defined as present in the evaluated lesions if the lesions were treated as progressive disease (defined by RANO), a predominant tumor was evident via biopsy, progressive worsening was evident by follow-up MRI within 8 weeks of the surveillance MRI or [11C]MET-PET scan, or the subject died of tumor progression without any other identifiable cause. Because all of the evaluated lesions were included at recurrence, OS was calculated from the date of diagnosis of recurrent tumor or pseudoprogression.

\section{Statistical analysis}

MRI and [11C]MET-PET readings were defined as true positives when definitely tumor scores correctly identified the final outcome and as false positives when definitely tumors scores differed from the final outcome. Ratings were defined as true negatives when definitely not tumor scores correctly identified the final outcome and as false negatives when definitely not tumor scores differed from the final outcome. Sensitivity and specificity 
were calculated by standard statistical definitions. Accuracy was defined as the proportion of true positives and true negatives in all of the scans. Interobserver agreement between different MRI and [11C]MET-PET observers was calculated with Cohen $\kappa$ values, which were interpreted as previously indicated (31). Log-rank tests were used to assess the association of subjective [11C]MET-PET and MRI findings with OS. By using optimal cutoff values, Kaplan-Meier curves were generated for MRI parameters (T1-enhancing volumes, FLAIR volumes), and PET parameter (SUVmax) to test whether these measurements from quantitative imaging analysis were associated with OS.

The sensitivity and specificity of metabolic tumor volume, TBR, T1 and FLAIR volumes and SUVmax using optimal cutoff values for predicting final outcomes were evaluated. We used the optimized cutoff values to categorize these imaging features, and log-rank tests were performed to test whether each of these features is associated with OS values, which calculated from the time of the MRI and [11C]MET-PET scans to the death of the subjects, or the date of the last follow-up up for alive subjects. The $95 \%$ confidence intervals for all diagnostic accuracy measures were calculated using bias-corrected bootstrap methods with resampling. All the statistical analyses were done using R Statistical Software.

\section{RESULTS}

We used May 2020 as the cutoff date for our analysis and found 27 patients who matched our inclusion criteria. We excluded 1 patient with L-2-hydroxyglutaric aciduria because differentiating tumor tissue from healthy brain was challenging beacuse of diffuse brain signal abnormalitiues in the entire brain due to this condition. Of the remaining 26 patients, 27 tumors ( 1 patient had a left frontal lobe recurrence that was treated and evaluated similarly to the original tumor in the cerebellum) were included in the analysis. Details of patient demographics and tumors are shown in Table 1 and Supplemetal Table 1. The details of the previous treatment, tumor location, and genetic alterations are included in Supplemetal Table 2. 


\section{Qualitative MRI and [11C]MET-PET interpretations for predicting final outcomes}

The final outcomes in 5 of the 27 lesions evaluated were no tumor present (i.e., pseudoprogression) and presence of tumor (i.e., tumor progression) in the remaining 22 lesions. The final outcomes were confirmed by follow-up MRI in 16 cases, by biopsy in 4, and by RANO criteria in 7.

The sensitivity, specificity, and accuracy of correctly predicting the presence of tumors from MRI by observer 1 were $86 \%$ (95\% CI: 64\% - 96\%), 80\% (95\% CI: 0 - 100\%), and 85\% (95\% CI: 63\% - 93\%), respectively, and 95\% (95\% CI: 73\% - 100\%),, 40\% (95\% CI: 0-100\%), and 85\% (95\% CI: 63\% - 93\%), respectively, for observer

2. The interobserver agreement was fair $($ Cohen $\kappa=0.49 ; P=0<.001)$. The sensitivity, specificity and accuracy for correctly predicting the presence of tumors by consensus readings were 95\% (95\% CI: 71\% - 100\%),, 60\% (95\% CI: 0 - 100\%), and 89\% (95\% CI: 67\% - 93\%), respectively. The details are summarized in Table 2.

The sensitivity, specificity, and accuracy for correctly predicting the presence of tumors with [11C]METPET scans were 100\% (95\% CI: NA),, 60\% (95\% CI: 0 - 100\%), and 93\% (95\% CI: 70\% - 96\%), respectively, and the interobserver agreement was $100 \%($ Cohen $\kappa=1)$. Positive [11C]MET-PET readings had higher sensitivity, specificity, and accuracy for correctly predicting presence of tumors than did individual MRI readings. [11C]MET-PET also had higher sensitivity, and accuracy for correctly predicting the presence of tumors than did the consensus MRI readings. The consesus MRI and [11C]MET-PET readings were concordant in $88.9 \%$ of cases and discordant in $11.1 \%$. In one subject, there was significant discprepancy between the MRI abnormality and PET abnormality, in which there were considerably surgery-related MRI abnormalities as the scans were obtained 21 days after surgery. (Fig 1).

We tested the accuracy between MRI observer 1, MRI observer 2, MRI consensus reads, and [11C]METPET reads in pairs with McNemar tests. There were no significant differences for any pair in the comparisons. In five of the 27 lesions, a discrepancy occurred between MRI observer 1, MRI observer 2, or the consensus MRI read for correctly predicting the final outcome, but [11C]MET-PET correctly predicted the final outcomes in all of these cases. The final outcomes of three of these five lesions were presence of tumor, and the final outcomes of 
two of these lesions were pseudoprogression. Only 1 case was indecisve for tumor versus nontumor treatmentrelated changes in the consensus MRI interpretation but was correctly predicted by the [11C]MET-PET evaluation (Fig 2).

\section{Quantitative imaging parameters from both [11C]MET-PET and MRI for predicting final outcomes}

The ROC curves of the SUVmax, metabolic tumor volume, TBRmax, TBRmean, T1 contrast enhancing tumor volume, and abnormal tumor volume by FLAIR were assessed for their ability to predict the final outcomes (32). ROC analysis yielded an optimal cutoff value of 3.3 for SUVmax to differentiate between the presence and absence of tumors $60 \%$ sensitivity (95\% CI: 36\% - 78\%), 100\% specificity(95\% CI: NA), and 67\% accuracy (95\% CI: (44\% - 81\%); $0.98 \mathrm{~cm}^{3}$ for metabolic tumor volume with a sensitivity of $90 \%$ (95\% CI: 69\%-100\%) and specificity of $80 \%(95 \%$ CI: $0 \%$ - 100\%), and accuracy 89\% (95\% CI: 64\% - 96\%); 1.82 for TBRmax with a sensitivity of $77 \%$ (95\% CI: 55\% - 91\%) and specificity of 100\%(95\% CI: NA), accuracy $81 \%$ (95\% CI: $59 \%$ 89\%), ; 1.4 for TBRmean with a sensitivity of $72 \%$ (95\% CI: 50\% - 88\%), specificity of 40\% (95\% CI: (0\% $100 \%)$, and accuracy 67\% (95\% CI: (44\% - 78\%), . The sensitivity, specificity, and accuracy of T1 contrastenhancing volumes of $2.4 \mathrm{~cm}^{3}$ or greater were $73 \%$ (95\% CI: $\left.50 \%-88 \%\right), 80 \%$ (95\% CI: (0\% - 100\%), and 74\% (95\% CI: 52\%, 85\%) respectively, and 86\% (95\% CI: 64\% - 96\%) and 80\% (95\% CI: 0 - 100\%), and $85 \%(95 \%$ CI: $63 \%-93 \%$ ), respectively, for abnormal FLAIR volumes of $13.76 \mathrm{~cm}^{3}$. The details are summarized in Table 2.

\section{Quantitative MRI and [11C]MET-PET interpretations associated with overall survival}

We used the optimized cutoff values to categorize imaging features, including the T1-enhancing volume, FLAIR volume, SUVmax, metabolic tumor volume, and TBR. T1 contrast enhancing tumor volume, metabolic tumor volume, TBR were significant by themselves for predicting final outcome. However, association of final outcome with the quantitative imaging parameters were not significant when tested with multivariable analysis. Log-rank tests were performed to test whether these imaging features are associated 
with OS. Using the above mentioned ROC-determined cut-off values, we found that there were that overall survival is significantly associated with metabolic tumor volume $(\mathrm{p}=0.0074)$, TBRmax $(\mathrm{p}=0.027)$, and T1 volume (p=0.016) (Fig. 3, 4). However, the SUVmax, TBRmean, and FLAIR volume did not show a significant association with OS.

\section{DISCUSSION}

Differentiating true tumor progression from treatment-related effects can be challenging because of overlapping features $(11,19,25)$. Many advanced MRI techniques and molecular imaging techniques have been studied to address this challenge $(19,25)$. Recent evidence suggests that amino acid PET tracers (i.e., [18]F- dihydroxyphenylalanine (F-DOPA)-PET and [18]F-Fluoroethyl Tyrosine (FET)PET) can assist conventional MRI at correctly identifying surgical margin and distinguishing between tumoral and nontumoral changes (15,33-36). [11C]MET-PET, in particular, has shown substantial promise (37-40), but these studies were only performed in adults, and many included metastatic nonprimary CNS tumors. Therefore, we explored the role of [11C]MET-PET in evaluating only recurrent PHGG.

The [11C]MET uptake is directly related to L-type amino acid transporter 1 (LAT1) expression (41); high [11C]MET uptake characteristically occurs in tumors with a high degree of neoangiogenesis and cellular proliferation $(8,41)$. Previous studies have demonstrated high sensitivity and specificity of [11C]MET-PET for diagnosing high-grade tumors $(8,42)$. In our study we found that the sensitivity and accuracy of $[11 \mathrm{C}]$ MET-PET for correctly differentiating true tumor progression from treatment-related effects are $100 \%$ and $93 \%$ respectively, compared to the reported $70-80 \%$ sensitivity and $75 \%$ accuracy in previous studies $(37,38,40)$. This difference may be due to the heterogeneous samples in the previous studies, which included both metastases and gliomas that were treated with different radiation doses and 
chemotherapy regimens. However, the sensitivity and specificity of the [11C]MET-PET for differentiating tumor progression from treatment related effects in our study is similar to the results of the study done by Dunkle et al (43). Our study also demonstrated higher specificity of the quantitative PET evaluation in comparison to qualitative evaluation. This is in contrast to the study done by Minamimoto et al. (37), which reported no significant difference between qualitative and quantitative [11C]MET-PET evaluations for assessment of tumor progression. More recently, study done by Marner et al has also demonstareted high specificity and accuracy of [18F]FET PET for differentiating tumor from non-tumor lesions (44).

Qualitative interpretation of MRI findings is the standard of care for follow-up of high-grade gliomas after treatment (19). Unlike qualitative [11C]MET-PET assessments, qualitative interpretation of MRI findings involves careful evaluation of many different MRI sequences that exploit the different magnetic properties of tissues and changes in these magnetic properties with MRI contrast compounds. This multifactorial evaluation process inherently leads to interpretation bias, as we observed in our study. The sensitivity, specificity, and accuracy of the 2 MRI observers in our study significantly differed, although both observers had expertise in evaluating pediatric brain tumors for ten years or more. Such interpretation bias influences the diagnostic performance of MRI; indeed, we found that the consensus MRI interpretation performed significantly better, similar to that of [11C]MET-PET, than did the individual MRI readings. Because consensus MRI interpretations by multiple neuroradiologists are not practical in routine clinical practice, the addition of [11C]MET-PET imaging of suspicious MRI findings adds value to the overall care of patients with PHGG.

Our study demonstrated significant association of metabolic tumor volume, and TBRmax with OS, as previously described $(45,46)$. Additionally, post contrast T1 volume was also significantly associated with OS, similar to multiple prior studies $(47,48)$. 
Our study includes limitations. The sample size was small but relatively large,considering the rarity of this tumor. As this study was initiated in 2009, the acqusition time of our PET scan is set to be 15 minutes, instead of currently recommended 20 minutes. In addition, the criteria for performing [11C]MET-PET on the included patients were based on a high clinical suspicion for recurrence or high likelihood of tumor recurrence on MRI findings. Consequently, there was a high pretest probability of the MRI abnormalities representing tumor recurrence, thereby introducing selection bias. A larger prospective multi-institutional study with regularly scheduled [11C]MET-PET scans might alleviate such selection bias. These studies should be sufficiently powered to examine whether [11C]MET-PET SUVmax cutoff values and qualitative interpretations can quantitatively predict final outcomes. However, due to the short half-life of carbon-11 ( 20 min), [11C]MET is currently available only at institutions with access to a cyclotron facility, such a study would need to be restricted to centers with [11C]MET synthesizing capability or institutions able to refer patients with suspicious findings on MRI to a center with [11C]MET synthesizing capability. To mitigate this problem, FET-PET with longer halflife is increasingly used in assessment of gliomas in many countries (49-52).

\section{CONCLUSION}

Our study shows that [11C]MET-PET has slightly higher sensitivity, specificity, and accuracy for correctly predicting the presence of tumor recurrence than does MRI when new or worsening imaging abnormalities are detected during surveillance imaging of previously treated PHGG. The interoberserver agreement of interpretation for [11C]MET-PET findings was excellent and better than that of MRI. Our study also shows that qunatitaive [11C]MET-PET and MRI can also predict overall survival. These findings indicate that [11C]MET-PET imaging may add value for predicting PHGG recurrence. However, the results from this small cohort should be validated in larger prospective, preferably multi-institutional studies. 
Grant Support:Author Melissa Wing worked in the project as a Pediatric Oncologic Edication (POE) traimning funded by National Institute of Health (\#R25CA23944). Additionally, this work was supported in part by the American Lebanese Syrian Associated Charities (ALSAC).

Financial Disclosure: AKB received an honorarium from ABC MedED, LLC, for MRI protocol optimization consulting. No other potential conflicts of interest relevant to this article exist.

Acknowldegement: We thank Dr. Amy Vavere, PhD for prduction of [11C]MET. We also thank Mitrea Bogdan, $\mathrm{PhD}$, for help in image segmentation and Nisha Badders, $\mathrm{PhD}$ for help in copyediting.

\section{KEY POINTS:}

QUESTION: How does the diagnostic perfomance of ${ }^{11} \mathrm{C}$-methionine PET compare with that of MRI for predicting tumors in lesions suspicious for recurrence during follow-up of pedatric high-grade gliomas?

ANSWER: [11C]MET-PET has 100\% sensitivity, $60 \%$ specificity, and $93 \%$ accuracy for correctly predicting the presence of tumors in new or worsening MRI abnormalities suspicious for tumors, in contrast with $95 \%, 60 \%$, and $89 \%$, respectively, for qualitative MRI interpretation. The interobserver agreement for [11C]MET-PET was higher than that for MRI.

IMPLICATIONS FOR PATIENT CARE: We found that ${ }^{11} \mathrm{C}$-methionine PET is a complementary modality to MRI for evaluating lesions suspicious of recurrence in previously treated pediatric highgrade glioma. 


\section{REFERENCES:}

1. Gajjar A, Bowers DC, Karajannis MA, Leary S, Witt H, Gottardo NG. Pediatric brain tumors: innovative genomic information is transforming the diagnostic and clinical landscape. J Clin Oncol 2015; 33(27): 2986-98.

2. Varlet P, Le Teuff G, Le Deley MC, et al. WHO grade has no prognostic value in the pediatric high-grade glioma included in the HERBY trial. Neuro Oncol 2020; 22: 116-27.

3. Reifenberger G, Wirsching HG, Knobbe-Thomsen CB, Weller M. Advances in the molecular genetics of gliomas implications for classification and therapy. Nat Rev Clin Oncol 2017; 14: 434-52.

4. Fangusaro J. Pediatric high grade glioma: a review and update on tumor clinical characteristics and biology. Front Oncol 2012; 2: 105.

5. Wolff JE, Driever PH, Erdlenbruch B, et al. Intensive chemotherapy improves survival in pediatric high-grade glioma after gross total resection: results of the HIT-GBM-C protocol. Cancer 2010; 116: 705-12.

6. Vern-Gross TZ, Schreiber JE, Broniscer A, Wu S, Xiong X, Merchant TE. Prospective evaluation of local control and late effects of conformal radiation therapy in children, adolescents, and young adults with high-grade glioma. Neuro Oncol 2014; 16: 1652-60.

7. Kline C, Felton E, Allen IE, Tahir P, Mueller S. Survival outcomes in pediatric recurrent high-grade glioma: results of a 20-year systematic review and meta-analysis. J Neurooncol 2018; 137: 103-10.

8. Garcia JR, Cozar M, Baquero M, et al. The value of (11)C-methionine PET in the early differentiation between tumour recurrence and radionecrosis in patients treated for a high-grade glioma and indeterminate MRI. Rev Esp Med Nucl Imagen Mol 2017; 36: 85-90.

9. Winter SF, Loebel F, Loeffler J, et al. Treatment-induced brain tissue necrosis: a clinical challenge in neuro-oncology. Neuro Oncol 2019; 21: 1118-30.

10. Reardon DA, Weller M. Pseudoprogression: fact or wishful thinking in neuro-oncology? Lancet Oncol 2018; 19: 1561-3.

11. Brandsma D, Stalpers L, Taal W, Sminia P, van den Bent MJ. Clinical features, mechanisms, and management of pseudoprogression in malignant gliomas. Lancet Oncol 2008; 9: 453-61.

12. Carceller F, Fowkes LA, Khabra K, et al. Pseudoprogression in children, adolescents and young adults with nonbrainstem high grade glioma and diffuse intrinsic pontine glioma. J Neurooncol 2016; 129: 109-21.

13. Langen KJ, Galldiks N, Hattingen E, Shah NJ. Advances in neuro-oncology imaging. Nat Rev Neurol 2017; 13: 279-89. 
14. Dhermain FG, Hau P, Lanfermann H, Jacobs AH, van den Bent MJ. Advanced MRI and PET imaging for assessment of treatment response in patients with gliomas. Lancet Neurol 2010; 9: 906-20.

15. Galldiks N, Dunkl V, Stoffels G, et al. Diagnosis of pseudoprogression in patients with glioblastoma using O-(2[18F]fluoroethyl)-L-tyrosine PET. Eur J Nucl Med Mol Imaging 2015; 42: 685-95.

16. Chu HH, Choi SH, Ryoo I, et al. Differentiation of true progression from pseudoprogression in glioblastoma treated with radiation therapy and concomitant temozolomide: comparison study of standard and high-b-value diffusion-weighted imaging. Radiology 2013; 269: 831-40.

17. Hu LS, Eschbacher JM, Heiserman JE, et al. Reevaluating the imaging definition of tumor progression: perfusion MRI quantifies recurrent glioblastoma tumor fraction, pseudoprogression, and radiation necrosis to predict survival. Neuro Oncol 2012; 14: 919-30.

18. Elshafeey N, Kotrotsou A, Hassan A, et al. Multicenter study demonstrates radiomic features derived from magnetic resonance perfusion images identify pseudoprogression in glioblastoma. Nat Commun 2019; 10: 3170.

19. Ellingson BM, Chung C, Pope WB, Boxerman JL, Kaufmann TJ. Pseudoprogression, radionecrosis, inflammation or true tumor progression? challenges associated with glioblastoma response assessment in an evolving therapeutic landscape. $J$ Neurooncol 2017; 134: 495-504.

20. Dankbaar JW, Snijders TJ, Robe PA, et al. The use of (18)F-FDG PET to differentiate progressive disease from treatment induced necrosis in high grade glioma. J Neurooncol 2015; 125: 167-75.

21. Maurer GD, Brucker DP, Stoffels G, et al. (18)F-FET PET imaging in differentiating glioma progression from treatmentrelated changes: a single-center experience. J Nucl Med 2020; 61: 505-11.

22. Hotta M, Minamimoto R, Miwa K. 11C-methionine-PET for differentiating recurrent brain tumor from radiation necrosis: radiomics approach with random forest classifier. Sci Rep 2019; 9: 15666.

23. Tan H, Chen L, Guan Y, Lin X. Comparison of MRI, F-18 FDG, and 11C-choline PET/CT for their potentials in differentiating brain tumor recurrence from brain tumor necrosis following radiotherapy. Clin Nucl Med 2011; 36: 978-81. 24. Kim YH, Oh SW, Lim YJ, et al. Differentiating radiation necrosis from tumor recurrence in high-grade gliomas: assessing the efficacy of 18F-FDG PET, 11C-methionine PET and perfusion MRI. Clin Neurol Neurosurg 2010; 112: 75865. 
25. Albert NL, Weller M, Suchorska B, et al. Response Assessment in Neuro-Oncology working group and European Association for Neuro-Oncology recommendations for the clinical use of PET imaging in gliomas. Neuro Oncol 2016; 18: 1199-208.

26. Takenaka S, Asano Y, Shinoda J, et al. Comparison of (11)C-methionine, (11)C-choline, and (18)F-fluorodeoxyglucosePET for distinguishing glioma recurrence from radiation necrosis. Neurol Med Chir (Tokyo) 2014; 54: $280-9$.

27. Lucas JT, Jr., Serrano N, Kim H, et al. (11)C-Methionine positron emission tomography delineates non-contrast enhancing tumor regions at high risk for recurrence in pediatric high-grade glioma. J Neurooncol 2017; 132: $163-70$. 28. Vavere A, Snyder SE (2012) Synthesis of L-[methyl-11C] methionine (11 C-MET). In: Scott PJ, Hockley B (eds) Radiochemical syntheses. Wiley, New York, pp 199-212

29. Law I, Albert NL, Arbizu J, Boellaard R, Drzezga A, Galldiks N, la Fougère C, Langen KJ, Lopci E, Lowe V, McConathy J, Quick HH, Sattler B, Schuster DM, Tonn JC, Weller M. Joint EANM/EANO/RANO practice guidelines/SNMMI procedure standards for imaging of gliomas using PET with radiolabelled amino acids and [18F]FDG: version 1.0. Eur J Nucl Med Mol Imaging. 2019;46:540-557.

30. Wen PY, Macdonald DR, Reardon DA, et al. Updated response assessment criteria for high-grade gliomas: response assessment in neuro-oncology working group. J Clin Oncol 2010; 28: 1963-72.

31. Landis JR, Koch GG. The measurement of observer agreement for categorical data. Biometrics 1977; 33: 159-74.

32. Vermont J, Bosson JL, Francois P, Robert C, Rueff A, Demongeot J. Strategies for graphical threshold determination. Comput Methods Programs Biomed 1991; 35: 141-50.

33. Galldiks N, Stoffels G, Filss C, et al. The use of dynamic O-(2-18F-fluoroethyl)-1-tyrosine PET in the diagnosis of patients with progressive and recurrent glioma. Neuro Oncol 2015; 17: 1293-300.

34. Herrmann K, Czernin J, Cloughesy T, et al. Comparison of visual and semiquantitative analysis of 18F-FDOPA-PET/CT for recurrence detection in glioblastoma patients. Neuro Oncol 2014; 16: 603-9.

35. Kebir S, Fimmers R, Galldiks N, et al. Late pseudoprogression in glioblastoma: diagnostic value of dynamic O-(2[18F]fluoroethyl)-L-Tyrosine PET. Clin Cancer Res 2016; 22: 2190-6.

36. Pirotte BJ, Lubansu A, Massager N, et al. Clinical impact of integrating positron emission tomography during surgery in 85 children with brain tumors. J Neurosurg Pediatr 2010; 5: 486-99.

37. Minamimoto R, Saginoya T, Kondo C, et al. Differentiation of brain tumor recurrence from post-radiotherapy necrosis with 11C-Methionine PET: visual assessment versus quantitative assessment. PLoS One 2015; 10: e0132515. 
38. Nihashi T, Dahabreh IJ, Terasawa T. Diagnostic accuracy of PET for recurrent glioma diagnosis: a meta-analysis. AJNR Am J Neuroradiol 2013; 34: 944-50, S1-11.

39. Felip-Falg s E, Teruel I, Domenech S, et al. C-Methionine PET (Met-PET) for a diagnosis algorithm for pseudoprogression (PsP), radiations necrosis $(\mathrm{RN})$ and progression $(\mathrm{P})$ after an indeterminate magnetic resonance imaging (MRI) in glioblastoma (GBM). Journal of Clinical Oncology 2018; 36(15_suppl): e14039-e.

40. Terakawa Y, Tsuyuguchi N, Iwai Y, et al. Diagnostic accuracy of 11C-methionine PET for differentiation of recurrent brain tumors from radiation necrosis after radiotherapy. J Nucl Med 2008; 49: 694-9.

41. Okubo S, Zhen HN, Kawai N, Nishiyama Y, Haba R, Tamiya T. Correlation of L-methyl-11C-methionine (MET) uptake with L-type amino acid transporter 1 in human gliomas. J Neurooncol 2010; 99: 217-25.

42. Grosu AL, Astner ST, Riedel E, et al. An interindividual comparison of O-(2-[18F]fluoroethyl)-L-tyrosine (FET)- and L[methyl-11C]methionine (MET)-PET in patients with brain gliomas and metastases. Int J Radiat Oncol Biol Phys 2011; 81: 1049-58.

43. Dunkl V, Cleff C, Stoffels G, et al. The usefulness of dynamic O-(2-18F-fluoroethyl)-L-tyrosine PET in the clinical evaluation of brain tumors in children and adolescents. J Nucl Med 2015; 56: 88-92.

44. Marner L, Lundemann M, Sehested A, Nysom K, Borgwardt L, Mathiasen R, Wehner PS, Henriksen OM, Thomsen C, Skjøth-Rasmussen J, Broholm H, Østrup O, Forman JL, Højgaard L, Law I. Diagnostic Accuracy and Clinical Impact of [ 18F]FET PET in Childhood CNS tumors. Neuro Oncol. E-pub ahead of print.

45. Van Dijken B, Ankrah A, Stormezand G, van Laar PJ, Dierckx R, van der Hoorn A. Prognostic value of 11C-methionine PET in patients with IDH wild-type glioma<strong/>. Journal of Nuclear Medicine 2019; 60(supplement 1): 1501.

46. Vigil C, Prieto E, Ribelles M, et al. Prediction of recurrence and survival analysis after radiotherapy of glioblastoma using 11C-Methionine PET/CT and MR. Journal of Nuclear Medicine 2014; 55(supplement 1): 12.

47. Huang RY, Rahman R, Hamdan A, et al. Recurrent glioblastoma: volumetric assessment and stratification of patient survival with early posttreatment magnetic resonance imaging in patients treated with bevacizumab. Cancer 2013; 119: 347988.

48. Ellingson BM, Kim HJ, Woodworth DC, et al. Recurrent glioblastoma treated with bevacizumab: contrast-enhanced T1weighted subtraction maps improve tumor delineation and aid prediction of survival in a multicenter clinical trial. Radiology 2014; 271: 200-10. 
49. Chan DL, Hsiao E, Schembri G, et al. FET PET in the evaluation of indeterminate brain lesions on MRI: Differentiating glioma from other non-neoplastic causes - A pilot study. J Clin Neurosci 2018; 58: 130-5.

50. Jena A, Taneja S, Gambhir A, et al. Glioma recurrence versus radiation necrosis: single-session multiparametric approach using simultaneous O-(2-18F-Fluoroethyl)-L-Tyrosine PET/MRI. Clin Nucl Med 2016; 41: e228-36.

51. Jeong SY, Lim SM. Comparison of 3'-deoxy-3'-[18F]fluorothymidine PET and O-(2-[18F]fluoroethyl)-L-tyrosine PET in patients with newly diagnosed glioma. Nucl Med Biol 2012; 39: 977-81.

52. Song S, Cheng Y, Ma J, et al. Simultaneous FET-PET and contrast-enhanced MRI based on hybrid PET/MR improves delineation of tumor spatial biodistribution in gliomas: a biopsy validation study. Eur J Nucl Med Mol Imaging 2020; 47: $1458-67$. 

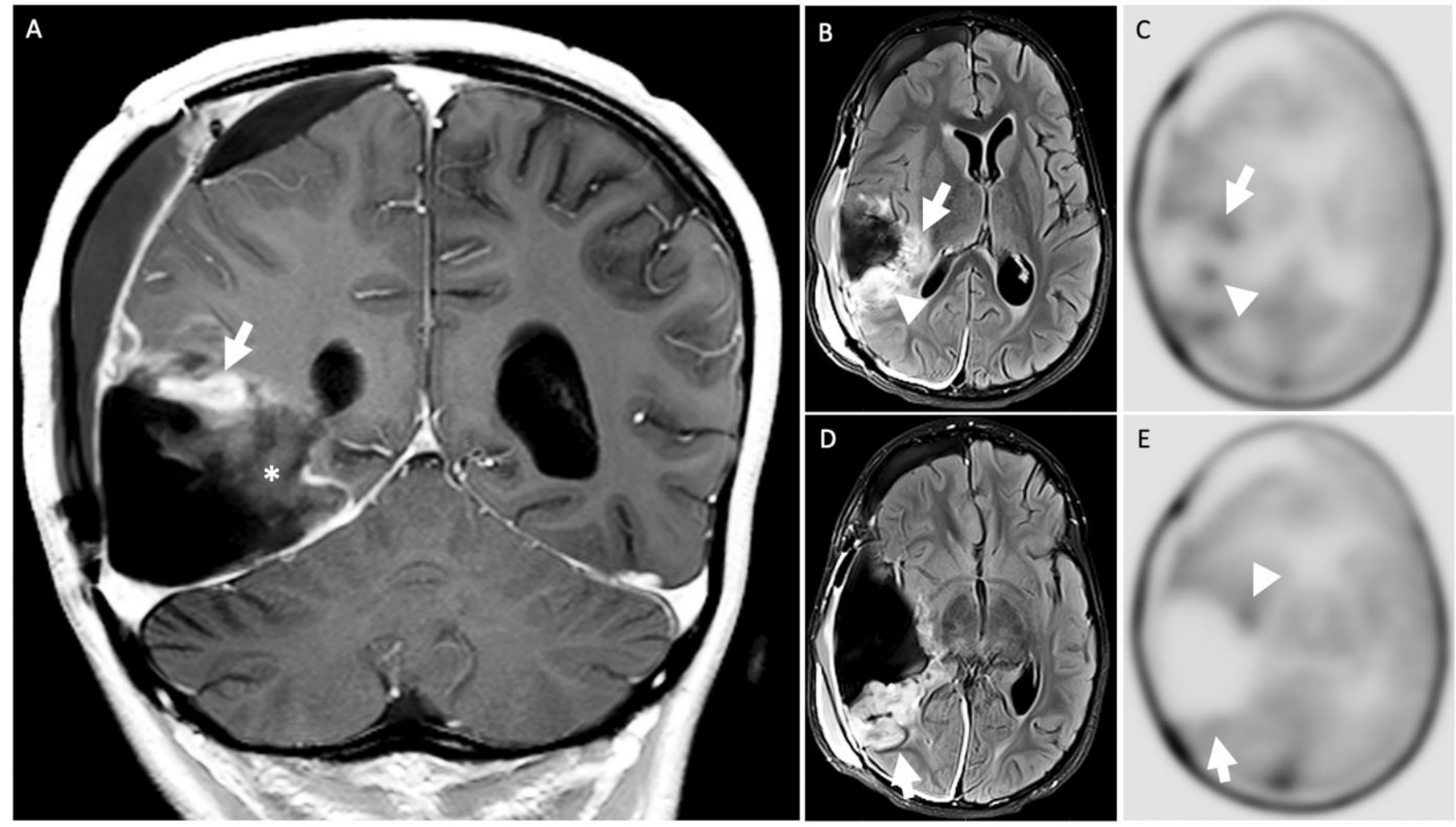

Figure 1. A. Post contrast coronal T1 weighted image demonstrates nodular enhancement (arrow) at the superior surgical margin. Images ( $\mathrm{B}$ and $\mathrm{C}$ correspond to the axial plane through this enhancement, whereas images $C$ and $D$ correspond to the axial plane demarcated by the *. T2 weighted image through the level of the basal ganglia shows a large cystic resection cavity in the right temporal lobe (white arrow). There is ill-defined T2 abnormality at the posterior aspect of the resection cavity (white arrow). B. Axial T2 FLAIR image obtained through the level of the nodular enhancement as seen on image A demonstrates areas of heterogeneously hyperintense tissue at the medial (white arrow) and posterior (arrowhead) surgical margin. C. Axial reconstruction of the MET-PET images through this level shows two foci of tracer uptake at the medial (arrowhead) and posterior (arrow) surgical margin. D. Axial T2 FLAIR image obtained through a plane inferior to the plane of image $\mathbf{B}$ and $\mathbf{C}$ demonstrates a relatively large areas of heterogeneously hyperintense tissue at the posterior surgical margin 
(white arrow). E. Axial reconstruction of the MET-PET images through this level shows no MET uptake at the posterior surgical margin (arrow). There is minimum uptake at the anteromedial surgical margin (arrowhead). Of note, this area was not included in the metabolic tumor volume due to low SUV (lower than 1.3 times the contralateral frontal lobe cortex). 

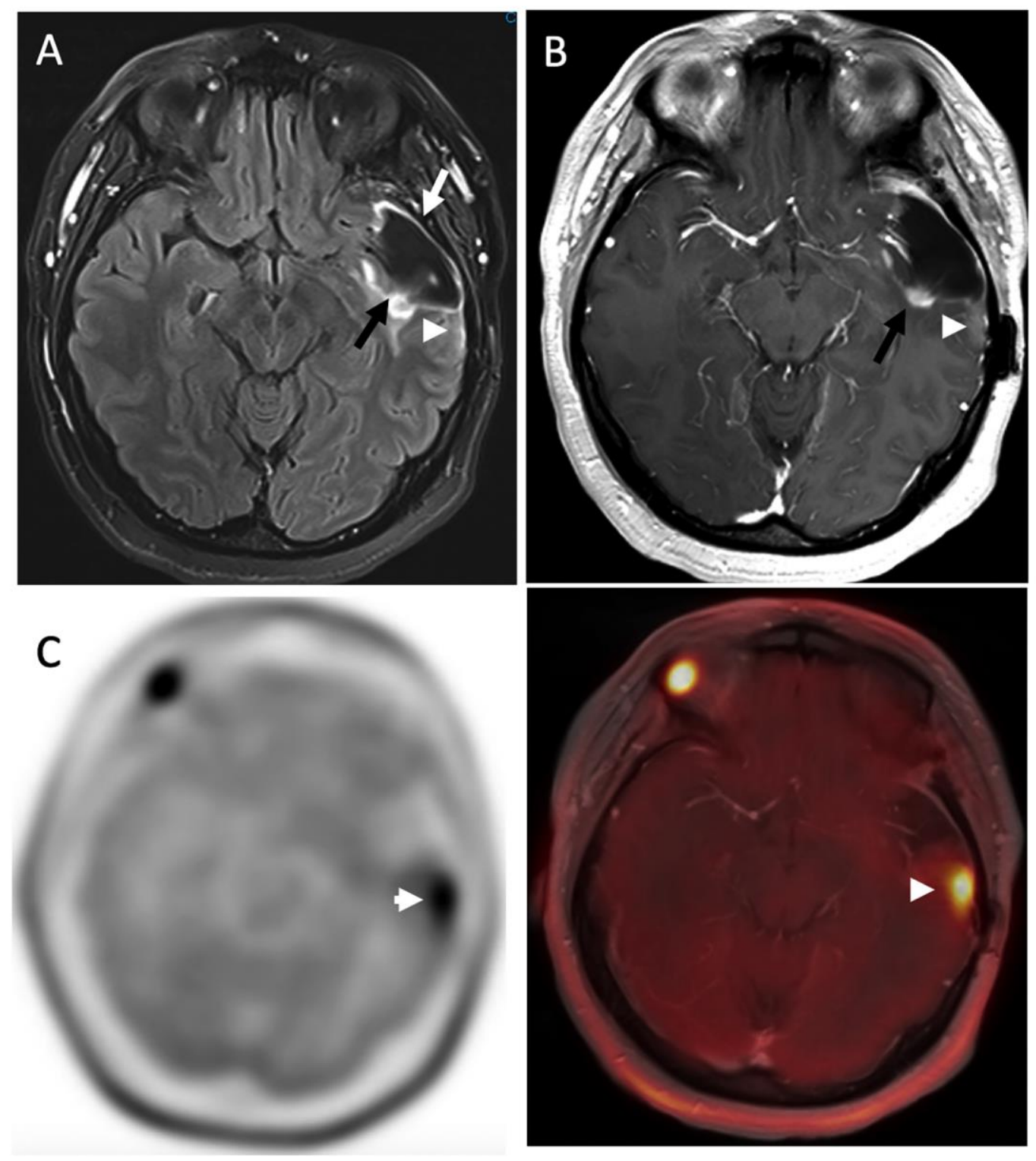

Figure 2. A. Axial T2 FLAIR weighted image through the level of midbrain shows a large cystic resection cavity in the left temporal lobe (white arrow). There is ill-defined T2 abnormality at the medial aspect of the resection cavity (black arrow). No obvious abnormality is noted posterior and lateral to the resection cavity (arrowhead). B. Axial post contrast T1-weighted image through the same level better shows focal area of contrast enhancement (black arrow). This enhancing focus has been followed up since prior treatment. Subtle contrast enhancement, a new finding compared to the previous MRIs, is noted posterior and lateral to 
the resection cavity (arrowhead). C. Axial reconstruction of the [11C]MET-PET images through the same level shows intense MET uptake posterior and lateral to the resection cavity (arrowhead) corresponding to the new subtle T1 enhancement. D. Postcontrast T1-MET-PET fused image also shows the MET abnormality corresponds to the new subtle enhancement at the posterior and lateral aspect of the resection cavity (arrowhead). 

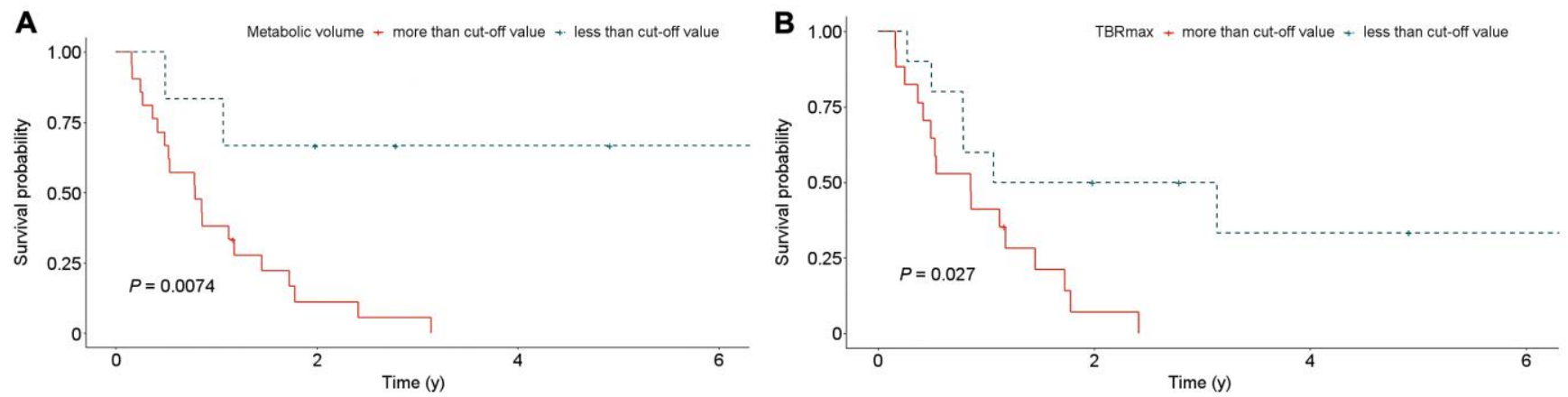

FIGURE 3. Kaplan-Meier curves demonstrating the overall survival probability of subjects according to MET-PET quantitative metrics. The $P$-values of the log-rank tests of the KaplanMeier curves for (A) metabolic tumor volume, and (B) TBRmax. 

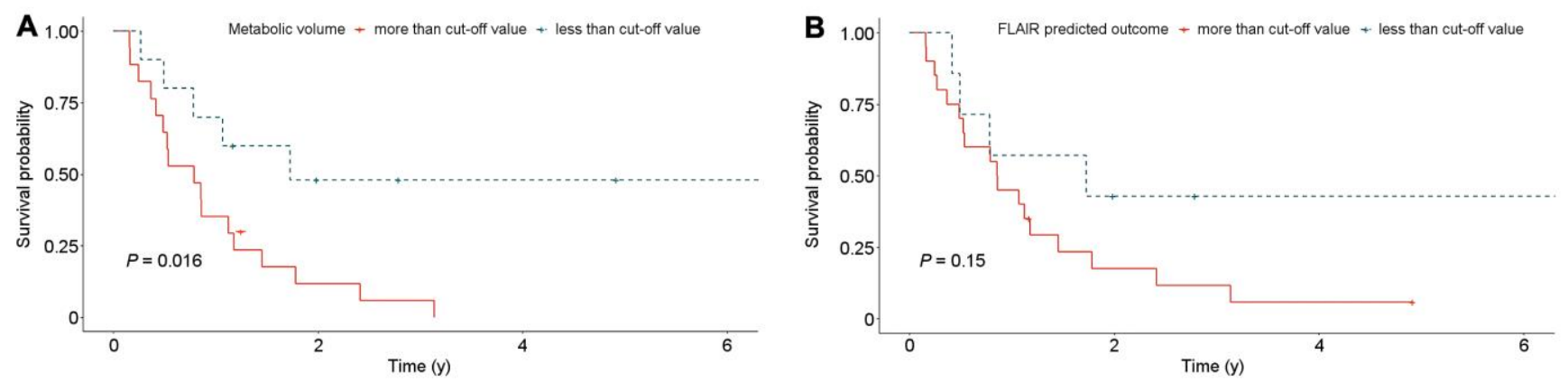

FIGURE 4. Kaplan-Meier curves demonstrating the overall survival probability of subjects according to quantitative MRI metrics. The $P$-values of the log-rank tests of the Kaplan-Meier curves for (A) Post contrast T1 volume and (B) FLAIR volume. 


\section{${ }^{11} \mathrm{C}-$ Methionine PET for Identification of Pediatric High-Grade Glioma Recurrence}

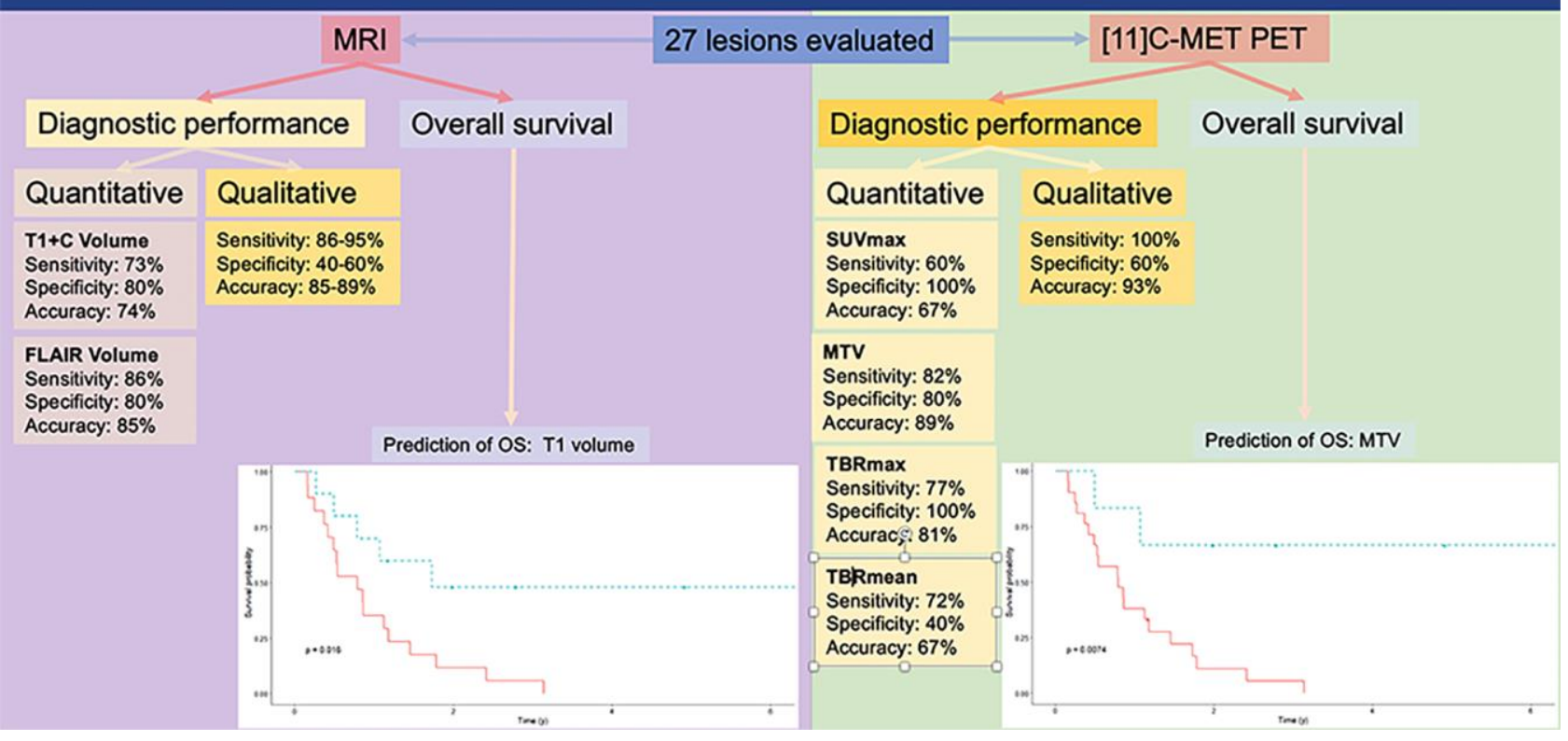

\section{Graphical Abstract}


TABLE 1 Demographcs of Patients Included in Study $(n=27)$

\begin{tabular}{|c|c|}
\hline Patient characteristics & No. patients \\
\hline \multicolumn{2}{|l|}{ Diagnosis } \\
\hline Glioblastoma & 17 \\
\hline WHO grade III astrocytoma & 5 \\
\hline High-grade neuroepithelial tumor & 2 \\
\hline High-grade glioma & 2 \\
\hline Anaplastic pleomorphic xanthoastrocytoma & 1 \\
\hline \multicolumn{2}{|l|}{ Age (years)* } \\
\hline $0-5$ & 4 \\
\hline $6-10$ & 2 \\
\hline $11-15$ & 8 \\
\hline $16-20$ & 8 \\
\hline $20-25$ & 4 \\
\hline \multicolumn{2}{|l|}{ Sex } \\
\hline Male & 16 \\
\hline Female & 10 \\
\hline \multicolumn{2}{|l|}{ Patient status } \\
\hline Deceased & 22 \\
\hline Alive & 4 \\
\hline
\end{tabular}

*Age at the time of PET imaging. 
Table 2. Diagnostic accuracy for tumor detection

\begin{tabular}{|l|l|l|l|l|l|l|l|l|}
\hline \multicolumn{9}{|c|}{ Diagnostic Accuracy for Tumor Detection } \\
\hline & $\begin{array}{l}\text { Qualitative } \\
\text { MRI } \\
\text { reading }\end{array}$ & $\begin{array}{l}\text { Qualitative } \\
\text { PET reading }\end{array}$ & $\begin{array}{l}\text { T1-enhancing } \\
\text { volume }\end{array}$ & $\begin{array}{l}\text { FLAIR } \\
\text { volume }\end{array}$ & SUVmax & MTV & TBRmax & TBRmean \\
\hline Sensitivity [CI] & $\begin{array}{l}0.95[0.71- \\
1]\end{array}$ & $1[$ na] & $\begin{array}{l}0.73[0.50- \\
0.88]\end{array}$ & $\begin{array}{l}0.86[0.64- \\
0.96]\end{array}$ & $\begin{array}{l}0.60[0.36- \\
0.78]\end{array}$ & $\begin{array}{l}0.90[0.69- \\
1]\end{array}$ & $\begin{array}{l}0.77[0.55- \\
0.91]\end{array}$ & $\begin{array}{l}0.72[0.50- \\
0.88]\end{array}$ \\
\hline Specificity [CI] & $0.60[0-1]$ & $0.60[0-1]$ & $0.80[0-1]$ & $0.80[0-1]$ & $1[$ na] & $0.80[0-1]$ & $1[\mathrm{na}]$ & $0.40[0-1]$ \\
\hline Accuracy [CI] & $0.89[0.67-$ & $\begin{array}{l}0.93[0.7- \\
0.96]\end{array}$ & $\begin{array}{l}0.74[0.52- \\
0.85]\end{array}$ & $\begin{array}{l}0.85[0.63- \\
0.93]\end{array}$ & $\begin{array}{l}0.67[0.44- \\
0.81]\end{array}$ & $\begin{array}{l}0.89[0.64- \\
0.96]\end{array}$ & $\begin{array}{l}0.81[0.59- \\
0.89]\end{array}$ & $\begin{array}{l}0.67[0.44- \\
0.78]\end{array}$ \\
\hline
\end{tabular}

Abbreviations: $\mathrm{Cl}$, Confidence interval; MRI, magnetic resonance imaging; MTV, metabolic tumor volume; PET,

[11C\}MET-PET; TBRmax, Tumor SUVmax to contralaetral normal brain SUVmean; TBRmean, Tumor SUVmean

to contralaetral normal brain SUVmean 
Supplemental Table 1. Detailed list of the qualitative and quantitative analyses of MET PET and MRI.

\begin{tabular}{|c|c|c|c|c|c|c|c|c|c|c|c|c|c|c|c|c|c|c|}
\hline $\begin{array}{l}\text { Lesions } \\
\text { Evaluated }\end{array}$ & $\begin{array}{l}\text { Age } \\
\text { (in Y) }\end{array}$ & $\begin{array}{l}\text { Time since } \\
\text { RT }\end{array}$ & $\begin{array}{l}\text { PET } \\
\text { Observer } \\
1^{\wedge}\end{array}$ & $\begin{array}{l}\text { PET } \\
\text { Observer } \\
2\end{array}$ & suVmax & TBRmax & $\begin{array}{l}\text { TBR } \\
\text { mean }\end{array}$ & $\begin{array}{l}\text { MTV } \\
\left(\mathrm{cm}^{3}\right)\end{array}$ & $\begin{array}{l}\text { Clin MR } \\
\text { Observer^^}\end{array}$ & $\begin{array}{l}\text { MRI } \\
\text { Observer } \\
1\end{array}$ & $\begin{array}{l}\text { MRI } \\
\text { Observer } \\
\mathbf{2}\end{array}$ & $\begin{array}{l}\text { Consensus } \\
\text { MR Grading }\end{array}$ & $\begin{array}{l}\text { GTV } \\
\text { FLAIR } \\
\left(\mathrm{cm}^{3}\right)\end{array}$ & $\begin{array}{l}\text { GTV } \\
\text { T1 } \\
\left(\mathrm{cm}^{3}\right)\end{array}$ & $\begin{array}{l}\text { Final } \\
\text { outcome }\end{array}$ & OS (in days) & $\begin{array}{l}\text { Diagnosis } \\
\text { by }\end{array}$ & $\begin{array}{l}\text { Tumor } \\
\text { type }\end{array}$ \\
\hline 1 & 24 & $>4$ weeks & 1 & 1 & 2 & 1.50 & 1.35 & 1.05 & 1 & 2 & 1 & 1 & 33.07 & 0.9 & 1 & 97 & MRI & GB \\
\hline 2 & 25 & $>4$ weeks & 1 & 1 & 3.5 & 1.60 & 1.4 & 0.98 & 1 & 2 & 1 & 1 & 25.33 & 0.54 & 1 & Alive & MRI & AA \\
\hline 3* & 16 & $>4$ weeks & 1 & 1 & 8.3 & 3.39 & 1.99 & 53.1 & 1 & 1 & 1 & 1 & 34.74 & 1.76 & 1 & 59 & MRI & GB \\
\hline $4^{*}$ & 15 & na & 2 & 2 & 1.9 & 1.46 & 0.65 & 0 & 3 & 2 & 3 & 3 & 4.52 & 1.74 & 2 & 179 & MRI & GB \\
\hline 5 & 9 & $>4$ weeks & 1 & 1 & 2.7 & 1.88 & 1.49 & 10.53 & 1 & 1 & 1 & 1 & 15.72 & 2.86 & 1 & 89 & Death & HGG \\
\hline 6 & 19 & $>4$ weeks & 1 & 1 & 2.4 & 1.79 & 1.43 & 3.9 & 1 & 1 & 1 & 1 & 86.28 & 2.45 & 1 & 287 & Biopsy & GB \\
\hline 7 & 25 & $>4$ weeks & 1 & 1 & 2.6 & 1.82 & 1.5 & 1.25 & 1 & 1 & 1 & 1 & 1.83 & 2.4 & 1 & 286 & MRI & GB \\
\hline 8 & 18 & $>4$ weeks & 1 & 1 & 6.2 & 3.13 & 1.71 & 39.48 & 1 & 1 & 1 & 1 & 110.3 & 2.71 & 1 & 313 & RANO & AA \\
\hline 9 & 23 & $>4$ weeks & 1 & 1 & 5.9 & 4.03 & 1.97 & 80.61 & 1 & 1 & 1 & 1 & 226.6 & 2.67 & 1 & 881 & RANO & OA \\
\hline 10 & 5 & $>4$ weeks & 2 & 2 & 0.9 & 0.65 & 0.63 & 0 & 3 & 2 & 3 & 2 & 2.38 & 0 & 2 & Alive & MRI & AA \\
\hline 11 & 5 & $>4$ weeks & 1 & 1 & 2.7 & 2.64 & 1.5 & 8.04 & 1 & 1 & 1 & 1 & 5.38 & 3.81 & 1 & 151 & MRI & GB \\
\hline 12 & 17 & $>4$ weeks & 1 & 1 & 6.7 & 3.59 & 1.67 & 76.33 & 1 & 1 & 1 & 1 & 79.11 & 58.33 & 1 & 58 & MRI & GB \\
\hline 13 & 7 & $>4$ weeks & 1 & 1 & 0.9 & 1.17 & 0.56 & 0 & 1 & 1 & 1 & 1 & 25.2 & 0.4 & 1 & 391 & Biopsy & GB \\
\hline 14 & 16 & $>4$ weeks & 1 & 1 & 4.3 & 2.75 & 1.58 & 55.92 & 3 & 1 & 1 & 1 & 58.12 & 28.19 & 1 & 411 & Biopsy & GB \\
\hline 15 & 13 & $>4$ weeks & 1 & 1 & 11.4 & 4.72 & 2.23 & 12.69 & 1 & 1 & 1 & 1 & 24.57 & 5.6 & 1 & 531 & RANO & HG PXA \\
\hline 16 & 12 & $>4$ weeks & 1 & 1 & 5.8 & 3.06 & 1.62 & 17.96 & 1 & 1 & 1 & 1 & 120.4 & 99.31 & 1 & 195 & MRI & HGG nos \\
\hline 17 & 14 & $>4$ weeks & 2 & 2 & 2 & 0.36 & 0.83 & 0 & 2 & 2 & 2 & 2 & 9.44 & 1.04 & 2 & Alive & MRI & HGNET \\
\hline 18 & 2 & $>4$ weeks & 1 & 1 & 2.1 & 2.21 & 1.53 & 9.5 & 1 & 1 & 1 & 1 & 53.14 & 7.52 & 1 & 314 & MRI & GB \\
\hline 19 & 12 & $>4$ weeks & 1 & 1 & 2.9 & 2.60 & 1.54 & 5.13 & 1 & 1 & 1 & 1 & 87.97 & 11.92 & 1 & 650 & RANO & GB \\
\hline 20 & 5 & ** & 1 & 1 & 0.9 & 1.81 & 1.28 & 11.27 & 1 & 1 & 1 & 1 & 69.03 & 68.92 & 2 & 1146 & Biopsy & HGNET \\
\hline 21 & 13 & $>4$ weeks & 1 & 1 & 2.9 & 1.47 & 1.37 & 0.18 & 2 & 2 & 2 & 2 & 4.76 & 2.12 & 2 & Alive & MRI & GB \\
\hline 22 & 19 & $>4$ weeks & 1 & 1 & 8.7 & 4.17 & 1.88 & 6.5 & 2 & 3 & 2 & 2 & 13.76 & 1.84 & 1 & 630 & MRI & GB \\
\hline 23 & 13 & ** & 1 & 1 & 9 & 6.24 & 2.01 & 136.8 & 2 & 1 & 1 & 1 & 176.8 & 93.03 & 1 & 134 & MRI & GB \\
\hline 24 & 12 & $<4$ weeks & 1 & 1 & 3.1 & 2.26 & 1.73 & 26.27 & 3 & 1 & 1 & 1 & 63.88 & 10.05 & 1 & 192 & MRI & GB \\
\hline 25 & 18 & $>4$ weeks & 1 & 1 & 5.8 & 3.54 & 1.99 & 133.3 & 1 & 1 & 1 & 1 & 113 & 1.77 & 1 & UK, lost follow-up & RANO & AA \\
\hline 26 & 17 & $<4$ weeks & 1 & 1 & 2.7 & 1.87 & 1.28 & 31.09 & 1 & 1 & 1 & 1 & 48.8 & 21.28 & 1 & 431 & RANO & GB \\
\hline 27 & 13 & $<4$ weeks & 1 & 1 & 6.6 & 4.22 & 1.86 & 289 & 1 & 1 & 1 & 1 & 133.7 & 3.37 & 1 & 178 & MRI & GB \\
\hline
\end{tabular}




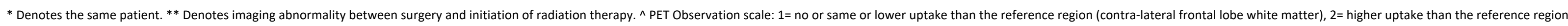

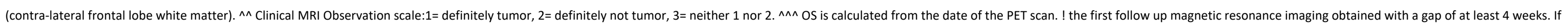

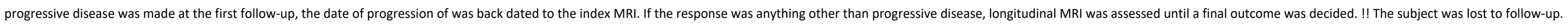

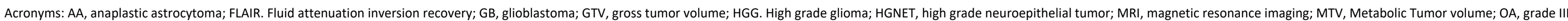

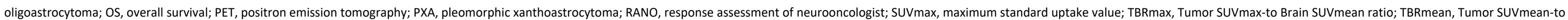
Brain SUVmean ratio; UK= unknown 


\section{Supplemental Table 2. Details of the tumors and the treatments}

\begin{tabular}{|c|c|c|c|c|c|}
\hline Lesion & Tissue Diagnosis & $\begin{array}{l}\text { Initial Tumor } \\
\text { Location }\end{array}$ & Treatment at Diagnosis & $\begin{array}{l}\text { Location of new } \\
\text { abnormality }\end{array}$ & Molecular markers \\
\hline 1 & Glioblastoma & $\mathrm{R}$ temporal lobe & Surgery+ RT with TMZ+ Follow-up TMZ & R temporal lobe & P53 mutation \\
\hline 2 & AA & $\mathrm{R}$ temporal lobe & Surgery+ RT with TMZ+ Follow-up TMZ and erlotinib & $\mathrm{R}$ temporal lobe & 19q13 deletion, IDH1 mutation \\
\hline 3 & Glioblastoma & Cerebellum & Surgery+ RT + Follow-up pembrolizumab & Cerebellum & $\begin{array}{l}\text { Alterations in PMS2, PIK3CA, FGFR1, } \\
\text { NF1, EZH2 }\end{array}$ \\
\hline 4 & Glioblastoma & $L$ frontal lobe & No treatment offered & L Frontal lobe & FGFR1 mutation, PIK3CA mutation \\
\hline 5 & HGNET & Cerebellum & Surgery+ RT + Follow-up COPE & Cerebellum & na \\
\hline 6 & Glioblastoma & $\mathrm{R}$ frontal lobe & $\begin{array}{l}\text { Surgery+ RT + Follow-up pembrolizumab; } 1 \text { recurrence treated with } \\
\text { Surgery+ RT with TMZ+ Follow-up TMZ+LOM }\end{array}$ & $R$ frontal lobe & P53 mutation \\
\hline 7 & Glioblastoma & L occipital lobe & Surgery+ RT with TMZ+ Follow-up TMZ+LOM, Multiple recurrences & corpus callosum & P53 mutation \\
\hline 8 & AA & Cerebellum & Surgery+ RT + Follow-up TMZ+Lomustine & Cerebellum & P53 mutation \\
\hline 9 & AA & Frontal lobes & Surgery+ RT + Follow-upL erlotinib; Multiple recurrences & L \& R Frontal lobes & na \\
\hline 10 & AA & L parietal lobe & Surgery +SJY07 chemotherapy & $\mathrm{R}$ frontal lobe & na \\
\hline 11 & Glioblastoma & Cerebellum & Surgery+ RT with TMZ+ Follow-up TMZ and Bev & Cerebellum & P53 mutation \\
\hline 12 & Glioblastoma & $\begin{array}{l}\text { R parietooccipital } \\
\text { lobes }\end{array}$ & Surgery+ RT with Vorinostat+ Follow-up TMZ+Bev & Both Frontal & CDKN2A, p53 \\
\hline 13 & AA & Thalamus & Biopsy + RT with TMZ + Follow-up with Etoposide & Thalamus & P53 mutation \\
\hline 14 & Glioblastoma & $\mathrm{R}$ parietal region & Surgery+ RT with TMZ+ Follow-up TMZ & $\mathrm{R}$ parietal region & P53 mutation, ATRX \\
\hline 15 & AA & L occipital lobe & Surgery+ RT with TMZ+ Multiple relapse and multiple therapy & L occiptal lobe & BRAFV600E \\
\hline 16 & HGG NOS & $\mathrm{R}$ thalamus & Biopsy + Carbo+VCR+ Multiple recurrences & $\mathrm{R}$ thalamus & H3.3 K27M \\
\hline 17 & HGNET & R parietal lobe & Surgery+ RT + Follow-up COPE & R parietal lobe & H3.3 K27M \\
\hline 18 & Glioblastoma & L frontal lobe & Surgery +SJY07 chemotherapy & $\mathrm{L}$ frontotemporal lobe & BCOR alteration \\
\hline 19 & Glioblastoma & $\mathrm{R}$ frontal lobe & Surgery+ RT with TMZ+ Follow-up TMZ+CCNU, multiple recurrences & $\mathrm{R}$ frontal lobe & P53 mutation \\
\hline 20 & HGNET & $\begin{array}{l}\mathrm{R} \text { frontal, temporal, } \\
\text { and parietal lobes }\end{array}$ & Surgery & $\begin{array}{l}\mathrm{R} \text { frontal, temporal, and } \\
\text { parietal lobes }\end{array}$ & BCOR mutation \\
\hline 21 & Glioblastoma & Cerebellum & Surgery+ RT with $T M Z+$ Follow-up $T M Z+C C N U$ & Cerebellum & ATRX \\
\hline 22 & Glioblastoma & L temporal lobe & Surgery $+\mathrm{RT}+$ Follow up with ribociclib and trametinib & L temporal lobe & P53 mutation, ATRX, 1q gain, CDKN2A \\
\hline 23 & Glioblastoma & $\mathrm{R}$ frontal lobe & Surgery & $\mathrm{R}$ frontal lobe & BRAFV600E \\
\hline 24 & Glioblastoma & Pons & Biopsy + RT & Pons & H3.3 K27M \\
\hline 25 & HGA NOS & $L$ frontal lobe & Surgery+ RT with TMZ+ Follow-up TMZ & $L$ frontal lobe & $\begin{array}{l}\text { H3F3A p.G34R, mutations in TP53 and } \\
\text { ATRX }\end{array}$ \\
\hline 26 & Glioblastoma & L temporal lobe & Surgery +RT with TMZ+ Follow-up TMZ & L temporal lobe & P53 mutation \\
\hline 27 & Glioblastoma & L parietal lobe & Surgery +RT with veliparib & L parietal lobe & H3F3A p.G34R \\
\hline
\end{tabular}

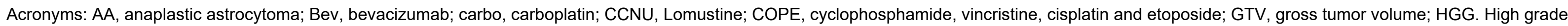

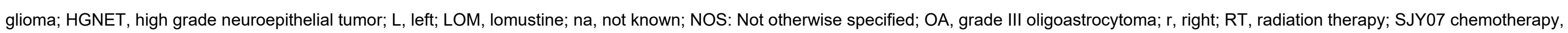
cyclophosphamide in combination with topotecan; TMZ, temozolomide; VCR, vincristine. 